\title{
The causal differential scattering approach to calculating the effective properties of random composite materials with a particle size distribution
}

A. Young and A. J. Mulholland and R.L. O'Leary

\begin{abstract}
An implementation of the Causal Differential Method (CDM) for modelling the effective properties of a random two-phase composite material is presented. Such materials are commonly used as ultrasonic transducer matching layers or backing layers. The method is extended to incorporate a particle size distribution in the inclusion phase. Numerical issues regarding the implementation and convergence of the method are discussed. It is found that, for a given frequency of excitation, the calculated velocity for the composite has a distribution whose variance increases as the volume fraction of inclusions increases. The model predictions would suggest that to reliably and repeatedly manufacture these composites, with a desired mechanical impedance, a low volume fraction of inclusions should be used.
\end{abstract}

\section{Introduction}

The transmission and detection of ultrasonic energy forms the basis for imaging and non-destructive testing systems in a broad range of applications such as biomedical therapy and diagnosis, underwater sonar, non-destructive testing, structural condition monitoring, industrial processing and control, and materials characterisation [4]. The requirement for efficient generation and detection over a desired frequency band is paramount and very often the limiting component of the entire system relates to the front end transducer design [7]. These devices normally consist of an active piezoelectric layer sandwiched between a backing material for damping

A. Young

University of Strathclyde, Department of Mathematics, Glasgow, UK

A. J. Mulholland

University of Strathclyde, Department of Mathematics, Glasgow, UK, e-mail: ajm@maths.strath.ac.uk

R.L. O'Leary

University of Strathclyde, Department of Electrical and Electronic Engineering, Glasgow 
and bandwidth control and some form of matching layer for interfacing to the mechanical load medium. Piezoelectric ceramics are mechanically stiff and do not interface well to media with low mechanical impedance such as water and air. Matching layers are therefore used to bridge this large impedance mismatch [5]. In many cases however these matching layers have to be manufactured by mixing two different materials in order to achieve the desired effective mechanical impedance [8]. A large variability in these effective properties can often be found for a given base and inclusion material, and volume fraction of inclusions. This is thought to be due to the random agglomeration of the inclusion particles to form percolation paths in the base material. This paper investigates this experimentally observed phenomenon by using the Causal Differential Method [3] with the addition of a particle size distribution to mimic the particle aggregation process. The model can be configured to assess the longitudinal and shear wave properties of the composite. This will allow for full characterisation of the material's elastic character. Such data is routinely employed in advanced two and three dimensional finite element analysis of these types of composite materials within transducer structures and as such a complete description of the elastic character is required. For example, matching layers are typically designed to a specific acoustic impedance in order to match the transducer to some load. However, in a transducer array configuration the matching layer is capable of supporting guided waves which will compromise the beam directivity and degrade imaging performance. Hence it is desirable to implement a methodology that will facilitate a more complete characterisation of these materials.

In the next section therefore, the model equations for a shear wave travelling in a two-dimensional plane containing randomly dispersed disc-like inclusions are presented. A dimensional rescaling is introduced to alleviate the numerical instabilities inherent to the model. A discussion on the convergence of the method and the effect that the properties of the two constituent materials have on the model's output is then given. Finally, the effect that the particle size distribution has on the variance of the model's output is presented.

\section{The Causal Differential Method}

By adding inclusions into a homogeneous material, the acoustic properties of the original material are modified by those of the inclusions [6]. In the regime where the particle size is commensurate with the wavelength of the insonifying wave there are a range of scattering theory methods for calculating the speed of propagation of the ultrasound wave [1]. The differential (or incremental) methods start by calculating the effective properties of a composite material containing a very small volume fraction of isolated particles (single particle scattering). The effective properties of this material are then used as the base material when adding another small volume fraction of inclusions. In this way the desired volume fraction of inclusions is obtained in a step by step manner and the final effective material properties are given by the final iteration. One such approach is the Causal Differential Method (CDM) [3]. 
The method considers the single scattering effect of adding small amounts of the inclusion, $\Delta \phi$, into a homogeneous matrix. By homogenising this new matrix, and repeating the process, the prescribed volume fraction of the inclusion, $\phi$, can be met. The predictions therefore lie between those given by a single-scattering approach and those given by a multiple-scattering method. The initial inclusion volume fraction is set at $\phi_{0}=\Delta \phi$ with $\rho_{0}$ (and $\mu_{0}$ ) set at the density (and shear modulus) of the pure base material. A subscript $I$ will be used to denote the corresponding properties of the inclusion material. The volume fraction is updated using

$$
\phi_{n+1}=\phi_{n}+\Delta \phi \quad n=0,1,2, \ldots, N-1 \quad,
$$

where $N$ is the number of steps in the iteration given by

$$
N=\frac{\phi}{\Delta \phi}
$$

The homogenised properties are then given by [2]

$$
\rho_{n+1}=\rho_{n}\left(1-\phi_{n}\right)+\rho_{I} \phi_{n}
$$

and

$$
\mu_{n+1}=\frac{\mu_{I}\left(1+\phi_{n}\right)+\mu_{n}\left(1-\phi_{n}\right)}{\mu_{I}\left(1-\phi_{n}\right)+\mu_{n}\left(1+\phi_{n}\right)} \mu_{n}
$$

The attenuation is calculated at each step using the additive law associated with acoustic attenuation given below by

$$
\alpha_{n+1}(\omega)=\alpha_{n}(\omega)+\Delta \phi \frac{\gamma_{n}^{e x}(\omega)}{2 \pi a^{2}}
$$

where $\pi a^{2}$ is the area of the inclusion. The extinction cross-section $\gamma_{n}^{e x}$ can be shown to simplify to the scattering cross-section of a single disc. This is given by the solution to the diffraction of elastic simple harmonic waves by an elastic embedded cylinder [3]. The incident wave is given by

$$
u_{z}^{i n}=e^{i\left(k_{n} x-\omega t\right)}
$$

and the scattered wave by

$$
u_{z}^{s c}=\sum_{m=0}^{\infty} A_{m} H_{m}^{(1)}\left(k_{n} r\right) \cos (m \theta) e^{-i \omega t},
$$

where $H_{m}^{(1)}$ is a Hankel function of the first kind of the $m^{\text {th }}$ order and the wave number $k_{n}$ of the current homogenised 'base' material is given by

$$
k_{n}=\frac{\omega}{c_{n}(\omega)}+i \alpha_{n}(\omega)
$$


The extinction cross-section $\gamma_{n}^{e x}$ is a function of $\omega, \rho_{n}, \rho_{I}, \mu_{n}, \mu_{I}$ and $a$ and is given by

$$
\gamma_{n}^{e x}=2\left(2\left|A_{0}\right|^{2}+\sum_{m=1}^{\infty}\left|A_{m}\right|^{2}\right)\left(k_{n}\right)^{-1}
$$

with

$$
\begin{aligned}
A_{m}= & -i^{m} \varepsilon_{m}\left(\begin{array}{c}
\mu_{I} k_{I} J_{m}\left(a k_{n}\right) J_{m}^{\prime}\left(a k_{I}\right) \\
-\mu_{n} k_{n} J_{m}^{\prime}\left(a k_{n}\right) J_{m}\left(a k_{I}\right)
\end{array}\right) \\
& /\left(\begin{array}{c}
\mu_{I} k_{I} H_{m}^{(1)}\left(a k_{n}\right) J_{m}^{\prime}\left(a k_{I}\right) \\
-\mu_{n} k_{n} H_{m}^{(1)^{\prime}}\left(a k_{n}\right) J_{m}\left(a k_{I}\right)
\end{array}\right)
\end{aligned}
$$

where

$$
\varepsilon_{m}=\left\{\begin{array}{ll}
1 & m=0 \\
2 & m \geq 1
\end{array},\right.
$$

and $J_{m}(z), J_{m}^{\prime}(z), H_{m}^{(1)}(z)$ and $H_{m}^{(1)^{\prime}}(z)$ denote the the $m^{\text {th }}$ order Bessel and Hankel functions of the first kind and their derivatives with respect to $z$, respectively, and the wave number in the inclusion material $k_{I}$ is given by

$$
k_{I}=\omega \sqrt{\frac{\rho_{I}}{\mu_{I}}}
$$

The phase velocity ignoring attenuation is given by

$$
v_{n}=\sqrt{\frac{\mu_{n}}{\rho_{n}}} .
$$

This value is then used to calculate the model's phase velocity adjusted for attenuation given by the Kramers-Kronig relationship

$$
c_{n+1}(\omega)=v_{n+1}\left(1+\frac{2 \omega^{2}}{\pi} v_{n+1} f_{0}^{\infty} \frac{\alpha_{n+1}(\Omega)}{\Omega^{2}\left(\Omega^{2}-\omega^{2}\right)} d \Omega\right)^{-1} .
$$

where the slash in the integral sign denotes Cauchy's principal value.

The algorithm is initialised by setting values for $\mu_{I}, \rho_{I}, \mu_{0}, \rho_{0}, a, \Delta \phi, \phi$, setting $\alpha_{0}=0$, setting $c_{0}=v_{0}$ using equation (13), and by setting $k_{I}$ using equation (12). The algorithm steadily updates the volume fraction using equation (1) until the desired volume fraction is achieved. At each step $k_{n}$ is calculated using equation (8), $A_{m}$ from equation (10), $\gamma_{n}^{e x}$ from equation (9), $\alpha_{n+1}$ from equation (5), $v_{n+1}$ from equations (4), (3) and (13), and finally $c_{n+1}$ from equation (14). The number of steps $N$ is given by equation (2) and so the phase velocity is given by

$$
c(\omega)=v_{N}\left(1+\frac{2 \omega^{2}}{\pi} v_{N} f_{0}^{\infty} \frac{\alpha_{N}(\Omega)}{\Omega^{2}\left(\Omega^{2}-\omega^{2}\right)} d \Omega\right)^{-1}
$$




\section{Numerical Implementation}

Four implementation issues present themselves:

- the inaccuracies and numerical instabilities associated with the very large and very small parameter values

- the singularity in the integrand of equation (14) and the associated Cauchy principal value

- the infinite integral in equation (14)

- the infinite summation in equation (9)

To improve the accuracy and efficiency of the computations a re-scaling of the dimensions was implemented. This allowed the program to compute with numbers of $O(1)$ instead of the extremely large and small values that exist. The fundamental units of mass (M), length (L) and time (T) were scaled by $\widehat{M}=\gamma M, \widehat{L}=\beta L$ and $\widehat{T}=\delta T$ with the scaling factors chosen to make each of the parameters as close as possible to $O(1)$. For the particular example considered here this was achieved by setting $\gamma=10^{6}, \beta=10^{3}$ and $\delta=10^{6}$ (see Table 1 ).

\begin{tabular}{|c|c|c|}
\hline Parameter & Dimensions & Scaling \\
\hline$\mu$ & $M L^{-1} T^{-2}$ & $\gamma \beta^{-1} \delta^{-2}$ \\
$\rho$ & $M L^{-3}$ & $\gamma \beta^{-3}$ \\
$a$ & $L$ & $\beta$ \\
$\omega$ & $T^{-1}$ & $\delta^{-1}$ \\
\hline$c, v$ & $L T^{-1}$ & $\beta \delta^{-1}$ \\
$k$ & $L^{-1}$ & $\beta^{-1}$ \\
$\gamma$ & $L$ & $\beta$ \\
$\alpha$ & $L^{-1}$ & $\beta^{-1}$ \\
\hline
\end{tabular}

Table 1 Dimensional Analysis of the Model Parameters

In equation (15) the integrand has a singularity at $\Omega=\omega$ and Cauchy's principal value takes the finite limit of the integral as $\Omega$ tends towards this singular point. This was numerically implemented by introducing a neighbourhood $\Delta \omega$ around this singularity. Additionally, a finite (but large) parameter $\omega^{u}$ was introduced to replace the infinite upper limit in this integral. A series of numerical experiments were then conducted to determine appropriate values for $\Delta \omega$ and $\omega^{u}$ that ensured reasonable convergence. A balance between the accuracy of the result with the computational time is then desired. A further numerical issue arose in the calculation of $\gamma_{n}^{e x}$ in equation (9) since the upper limit in the summation is infinite. This was replaced by a finite sum to $M$ terms. In order to apply the model there are therefore four implementation parameters that need to be set: $M, \Delta \phi, \Delta \omega$ and $\omega^{u}$. Convergence was assessed by considering the summed difference in the phase velocity over a suitable angular frequency range defined as 


$$
F\left(M, \Delta \phi, \Delta \omega, \omega^{u}\right)=\sum_{i}\left|c^{j+1}\left(\omega_{i}\right)-c^{j}\left(\omega_{i}\right)\right|^{2},
$$

where $j$ represents the $j^{t h}$ value of the particular parameter being examined. For this analysis three of the parameters were fixed, and the decay in $F$ examined whilst the fourth parameter was varied. When varying $\omega^{u}$, the phase velocity had a range $\omega \in\left[1.7 \times 10^{3}, 2.5 \times 10^{5}\right] \mathrm{Hz}$ with the remaining numerical parameters fixed at $M=3, \Delta \phi=4.625 \times 10^{-2}(N=4)$ and $\Delta \omega=15 \mathrm{~Hz}$. The physical parameters were chosen similar to those in [3]: $\phi=0.185, \mu_{I}=0.1 \mathrm{GPa}, \mu_{0}=$ $0.0155 \mathrm{GPa}, \rho_{I}=1 \times 10^{3} \mathrm{Kg} \mathrm{m}^{-3}, \rho_{0}=1.075 \times 10^{3} \mathrm{Kg} \mathrm{m}^{-3}$ and $a=2.3 \mathrm{~mm}$. The results were compared for $\left(\omega^{u}\right)^{j}=\{0.2,0.8,1.7,10,18\} \times 10^{5} \mathrm{~Hz}$ respectively. The results showed that $F$ converged to a reasonable level for $\omega^{u} \geq 0.8 \times 10^{5} \mathrm{~Hz}$ with a linear relation between the computational time and $\left(\omega^{u}\right)^{j}$. This procedure was repeated in order to determine the effect of increasing $M$. All parameters were identical with the exception of fixing $\omega^{u}=0.8 \times 10^{5} \mathrm{~Hz}$ and running the model with $(M)^{j}=\{1,3,5,7,10,15,20\}$. Again $F$ converged as $M$ increased, a linear increase in computational time was observed and reasonable convergence was observed for $M \geq 5$. A similar analysis was conducted for $\Delta \omega$ however, unlike $\omega^{u}$ and $M$, there was no linear relationship between the computational time and $\Delta \omega$. The effect of increasing $N$ (decreasing $\Delta \phi$ ) was analysed over different volume fractions $\phi$ for the same angular frequency range with $M=9$ and $\omega^{u}$ as above. The volume fractions considered were $\phi^{j}=\{0,0.25,0.5,0.75,1\}$ with $N^{j}=\{2,4,6 \ldots 18,20\}$. It was observed that there was a linear increase in the computational time as $N$ increased, as expected. There was also evidence of a linear increase in the computational time as the angular frequency increased. Additionally, the phase velocity tended to a limit as $N$ increased and if too large a value for $\Delta \phi$ was chosen then the method became unstable.

\section{Analysis of Relationships of Physical Properties in Model}

In this section the model was applied to a material commonly used to produce transducer matching layers and backing blocks (epoxy resin and tungsten particles). The appropriate data are summarised in Table 2.

\begin{tabular}{|l|c|c|}
\hline Material & $\mu(\mathrm{G} \mathrm{Pa})$ & $\rho\left(\mathrm{Kgm}^{-3}\right)$ \\
\hline Epoxy & 1.48 & 1140 \\
Tungsten & 161.2 & 19300 \\
\hline
\end{tabular}

Table 2 Physical properties of epoxy and tungsten

The effect that the various physical parameters of the two constituents have on the model's prediction of the phase velocity was then examined. For example, it 
was found that the phase velocity increased as the square root of the effective shear modulus and as the square root of the effective density. The radius of the inclusions has a marked influence on the final phase velocity. The typical mean particle size used for the tungsten inclusions was $4-6 \mu \mathrm{m}$ with a range of other commercially available mean particle sizes being $a=\{1,10,25,100,250\} \mu \mathrm{m}$.

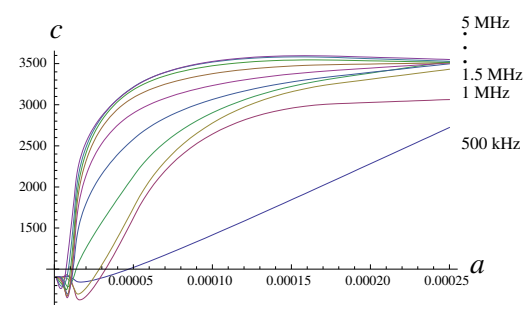

Fig. 1 Phase velocity $c\left(\mathrm{~ms}^{-1}\right)$ versus particle size $a(\mathrm{~m})$ for frequencies $500 \mathrm{kHz}$ to $5 \mathrm{MHz}$, with inclusion volume fraction $\phi=0.3$. The velocity profiles move upwards monotonically as the frequency increases.

Figure 1 shows that the velocity changes as the particle size increases and that it does so in a nonlinear fashion. In addition, at these values of the particle size, this velocity depends on the frequency of the ultrasound wave. In this region the particle size is commensurate with the wavelength of the ultrasound wave. As the frequency increases the velocity profile increases monotonically until once again the velocity curves converge for very high frequencies.

\section{Comparison between the theoretical predictions and experimental measurements}

In order to carry out an experimental validation of this model a number of samples of epoxy resin, filled with tungsten particles, were prepared and their acoustic properties measured by a through transmission method [9]. The epoxy resin, CY1303/HY1300, was supplied by Huntsman, Cambridge, UK and the tungsten particles with a mean particle size of $5 \mu \mathrm{m}$ were supplied by Ultimate Metals, Chingford, UK. The epoxy resin was mixed by hand in accordance with the manufacturer's instructions and degassed in a vacuum chamber to remove any entrained air bubbles. The epoxy and tungsten, in the required volume ratio, were placed in a pestle and mortar and the mixture carefully ground together. For each sample sufficient material was prepared in excess to produce a sample with the dimensions $49 \mathrm{~mm}$ diameter with a $10 \mathrm{~mm}$ thickness. Once thoroughly mixed the material were transferred to a mould with a close fitting lid. The mould was designed to allow the excess material to escape prior to sealing the mould, once sealed the tungsten-epoxy composite was allowed to cure for 18 hours at room temperature prior to being re- 
moved from the mould. Once removed from the mould the major faces were then machined to ensure parallelism for the measurement of acoustic properties.

In samples with low volume fraction, in the current context $\phi \leq 0.4$, the density of the tungsten particles will mean that settlement will occur during the cure cycle. To limit the settlement, the cylindrical mould is mounted axially into a rotation fixture and rotated about the axis during the cure cycle. In the case of samples where $\phi \geq 0.4$, the tungsten-epoxy composite must be cured under pressure in order to obtain a uniform fully cured composite. To apply pressure to the curing material a reusable cylindrical mould manufactured from PTFE was used, a simple plunger mechanism was incorporated into the mould design such that the material could be compressed using a hydraulic press attached to the plunger. For each sample having a volume fraction greater than 0.4 , the mould was subject to 1000 psi pressure during the cure cycle.

The acoustic velocities were measured using a through transmission time of flight method. Since the samples were acoustically matched to water, oil having a velocity of $680 \mathrm{~m} / \mathrm{s}$ was employed as the coupling medium in order to refract a shear wave into the sample. Figure 2 shows the measured acoustic velocities and these compare well with those obtained using the theoretical approach detailed in Section 2.

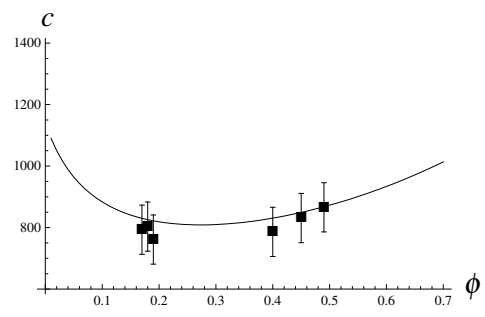

Fig. 2 Phase velocity $c\left(\mathrm{~ms}^{-1}\right)$ versus the volume fraction of the tungsten inclusions $\phi$ at frequency $500 \mathrm{KHz}$. The model results (full line) are compared with experimentally measured values (filled squares), with a particle size of $5 \mu \mathrm{m}$.

\section{A Particle Size Distribution}

In a practical situation it is impossible to add inclusions of identical size. In fact, most commercially available materials follow a normal distribution with a specified mean particle size. It is also of interest to investigate the root cause for the experimentally observed variations in the measured mechanical impedance of epoxytungsten samples at a fixed volume fraction of inclusions. The CDM algorithm was adapted by replacing the fixed particle size at each step with a random value drawn from a normal distribution of known mean and standard deviation. The phase velocity was plotted as a function of the final volume fraction of inclusions with the 
physical parameters set at those for epoxy and tungsten (see Table 2), with $\bar{a}=5 \mu \mathrm{m}$, and the implementation parameters set as $M=9, \Delta \phi=0.05$ and $\Delta \omega=0.8 \times 10^{3} \mathrm{~Hz}$. The process was conducted for a standard deviation of $\sigma_{1}=0.1 \bar{a}$ to determine the effect that a spread of particle sizes in a sample of tungsten powder would have. The algorithm was run several times and the variation in the results was then compared with those from a fixed particle size simulation.

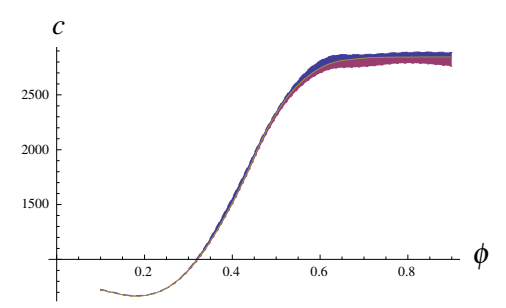

Fig. 3 Phase velocity $c\left(\mathrm{~ms}^{-1}\right)$ versus inclusion volume fraction $\phi$ at frequency $f=1 \mathrm{MHz}$ for random particle size selection from a normal distribution with $\bar{a}=5 \mu \mathrm{m}$ and a $10 \%$ standard deviation. The shaded regions denote the variance in the model's output lying above and below the fixed particle size simulation.

Figures 3 shows an increase in the difference between the maximum and the minimum values from the phase velocity for constant particle size as the volume fraction increases.

An alternative view can be taken of these results by fixing the volume fraction ( $\phi=0.2$ and $\Delta \phi=0.04$ ) and calculating the phase velocity as the frequency of the insonifying wave is varied.

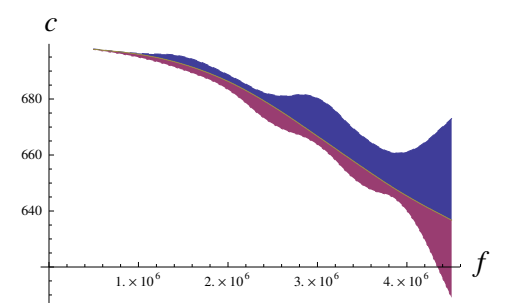

Fig. 4 Phase velocity $c\left(\mathrm{~ms}^{-1}\right)$ versus frequency $f(\mathrm{~Hz})$ at an inclusion volume fraction of $\phi=0.2$, for a random particle size selection from a normal distribution with $\bar{a}=5 \mu \mathrm{m}$ and a $10 \%$ standard deviation. The shaded regions denote the variance in the model's output lying above and below the fixed particle size simulation.

As the frequency increases, the variance in phase velocity due to the random particle size increases, highlighting the fact that the higher the frequency the more the variations in particle size affect the velocity. In order to produce a composite for a transducer matching layer, capable of working over a broad-bandwidth, it is important to have a sample of inclusions with as low a variance as possible in a size regime 
that is outwith the effects of scattering. This will allow a greater predictability of the phase velocity, and as a result be able to produce a desired composite material with greater control.

\section{Conclusion}

Ultrasonic transducer designs typically consist of an active piezoelectric layer sandwiched between a backing material for damping and bandwidth control and some form of matching layer for interfacing to the mechanical load medium. These materials are very often manufactured by mixing two different materials in order to achieve a desired mechanical impedance. A large variability in these effective properties can often be found for a given base and inclusion material, and a fixed volume fraction of inclusions. This is thought to be due to the random agglomeration of the inclusion particles to form percolation paths in the base material. In this paper an implementation of the Causal Differential Method (CDM) has been presented and, by incorporating a particle size distribution in the inclusion phase, the root cause of this variability in the effective composite properties is examined. Numerical issues regarding the implementation and convergence of the method were also discussed. Reasonable convergence and computational times for the method were found as functions of the implementation parameters in the model. A dimensional rescaling was also introduced to alleviate the numerical instabilities inherent to the model. It was found that, for a given frequency of excitation, the calculated velocity for the composite has a distribution whose variance increases as the volume fraction of inclusions increases. This would suggest that to reliably and repeatedly manufacture composites with a desired mechanical impedance, a low volume fraction of inclusions should be used.

\section{References}

1. Anson, L.W., Chivers, R.C.: Ultrasonic Velocity in Suspensions of Solids in Solids - A Comparison of Theory and Experiment. J. Physics D: Appl. Phys. 26, 1566-1575 (1993)

2. Beltzer, A.I.: Acoustics of Solids. Springer-Verlag, Berlin (1988)

3. Beltzer, A.I., Brauner, N.: The Dynamic Response of Random Composites by a Causal Differential Method. Mech. of Mats. 6, 337-345 (1987)

4. Beyer, R.T., Letcher, S.V.: Physical Ultrasonics. Academic Press, New York (1969)

5. Desilets, C.S., Fraser, J.D., King, G.S.: The design of efficient broad-band piezoelectric transducers. IEEE Trans. Sonics. Ultrasonics SU-25(3), 115-125 (1978)

6. Grewe, M.G., Gururaja, T.R., Shrout, T.R., Newnham, R.E.: Acoustic Properties of Particle/Polymer Composites for Transducer Backing Applications. IEEE Trans. UFFC. 37(6), 506-514 (1990)

7. Hayward, G.: The influence of pulser parameters on the transmission response of piezoelectric transducers. Ultrasonics 23(3), 103-112 (1985) 
8. Mulholland, A.J., Ramadas, N., O’Leary, R.L., Parr, A., Troge, A., Pethrick, R.A., Hayward, G.: Enhancing the Performance of Piezoelectric Ultrasound Transducers by the use of Multiple Matching Layers. IMA J. Appl. Maths. (Accepted), (2008)

9. O'Leary, R.L.: An investigation into the passive materials utilized in the construction of piezoelectric composite transducers. Ph.D. thesis, University of Strathclyde, Glasgow, (2003) 